\title{
The Role of Spin Anisotropy in the Unbinding of Interfaces
}

\author{
C. Micheletti and J. M. Yeomans \\ Department of Physics, Theoretical Physics, University of Oxford, \\ 1 Keble Road, Oxford OX1 3NP, U.K.
}

\begin{abstract}
We study the ground state of a classical X-Y model with $p \geq 3$-fold spin anisotropy $D$ in a uniform external field, $H$. An interface is introduced into the system by a suitable choice of boundary conditions. For large $D$, as $H \rightarrow 0$, we prove using an expansion in $D^{-1}$ that the interface unbinds from the surface through an infinite series of layering transitions. Numerical work shows that the transitions end in a sequence of critical end points.
\end{abstract}

PACS numbers: 0550; 6470; 7540D

Typeset using REVTEX 
When an interface unbinds from a surface it can do so through a first or second order transition or, on a lattice, via a sequence of first order layering transitions. In general the nature of the unbinding, or wetting, transition depends on the details of the microscopic interactions and external parameters such as the temperature and applied magnetic field [1, 2].

In a discrete spin model at zero temperature long-range interactions are needed to stabilise series of first order layering transitions. However, if the interactions are short range, an infinite sequence of such transitions can occur at finite temperatures [3]. This results from competition between the localising effect of the binding potential and the entropic terms in the free energy which favour a delocalised interface.

In this paper we aim to discuss the role of a hithertofore unexplored parameter on the unbinding transition: the spin anisotropy. We shall show that, as discrete spins soften, layering transitions can be stabilised in simple, short-range clock models, even at zero temperature. An expansion in inverse spin anisotropy allows us to prove that an infinite sequence of layering phase transitions exist. Moreover, because the interesting features occur at zero temperature it is possible to follow the phase diagram numerically for all values of the spin anisotropy. In particular we are able to demonstrate how the boundaries between the different interface phases end in critical end points and to pinpoint these with considerable precision.

We consider the classical $\mathrm{X}-\mathrm{Y}$ model in a magnetic field $H$ with $p$-fold anisotropy $D$. The model is defined by the Hamiltonian

$$
\mathcal{H}=\sum_{i=1}^{N}\left\{-J \cos \left(\theta_{i-1}-\theta_{i}\right)-H\left(\cos \theta_{i}-1\right)-D\left(\cos p \theta_{i}-1\right) / p^{2}\right\}
$$

where $i$ labels the spins on a one-dimensional lattice and $\theta_{i}$ can take values between 0 and $2 \pi$. An interface is imposed on the system by choosing $\theta_{0}=2 \pi / p$, and $\theta_{N}=0$ and letting $N \rightarrow \infty$. If the interface lies to the right of the $n^{\text {th }}$ spin from the end of the chain (that is, for $\theta_{0}$ fixed, between $i=n-1$ and $i=n$ ) the corresponding interface phase will be labelled $\langle n\rangle$.

For infinite $D$ the Hamiltonian (11) describes a $p$-state clock model. For $H>0$ the 
interface is bound to the surface in state $\langle 1\rangle$. The point $(H=0, D=\infty)$ is a multiphase point where the interface has the same energy whatever its position on the lattice. As the anisotropy is reduced from infinity it becomes, for $p \geq 3$, energetically favourable for the spins to relax from their clock positions. This results from competition between the field term, which favours an interface in position $\langle 1\rangle$ and the exchange interaction which prefers to minimise the angle between the spins and hence favours a free interface. The result of this is that the degeneracy at the multiphase point is broken and the interface unbinds from the surface through an infinite series of first order layering transitions $\langle 1\rangle,\langle 2\rangle,\langle 3\rangle \ldots$.

For large $D$ the existence of these transitions can be proven using an expansion in $D^{-1}$ 逃. Writing

$$
\theta_{i}=\theta_{i}^{0}+\tilde{\theta}_{i}
$$

where $\theta_{i}^{0} \equiv \theta_{i}(D=\infty)$, and keeping only terms quadratic in the angles and their differences the Hamiltonian (1) becomes

$$
\begin{gathered}
\mathcal{H}=\sum_{i=1}^{\infty}\left\{-J c_{i}-H \cos \left(\theta_{i}^{0}-1\right)+J c_{i}\left\{\tilde{\theta}_{i-1}-\tilde{\theta}_{i}+s_{i} / c_{i}\right\}^{2} / 2-J s_{i}^{2} / 2 c_{i}+\right. \\
\left.H \cos \theta_{i}^{0}\left\{\tilde{\theta}_{i}+\tan \theta_{i}^{0}\right\}^{2} / 2-H \sin ^{2} \theta_{i}^{0} / 2 \cos \theta_{i}^{0}+D \tilde{\theta}_{i}^{2} / 2\right\}
\end{gathered}
$$

where

$$
s_{i}=\sin \left(\theta_{i-1}^{0}-\theta_{i}^{0}\right), \quad c_{i}=\cos \left(\theta_{i-1}^{0}-\theta_{i}^{0}\right) .
$$

The equilibrium values of the $\tilde{\theta}_{i}$ are given by minimising the Hamiltonian (3). This leads to linear recursion equations

$$
-J c_{i}\left\{\tilde{\theta}_{i-1}-\tilde{\theta}_{i}+s_{i} / c_{i}\right\}+J c_{i+1}\left\{\tilde{\theta}_{i}-\tilde{\theta}_{i+1}+s_{i+1} / c_{i+1}\right\}+H \cos \theta_{i}^{0}\left(\tilde{\theta}_{i}+\tan \theta_{i}^{0}\right)+D \tilde{\theta}_{i}=0 .
$$

If the full Hamiltonian (1) is used non-linearities appear in the recursion relations (5). However, these do not affect the leading order terms needed for the subsequent calculations.

Leading order corrections to the values of the spins can easily be read off from the recursion equations (5). For the phase $\langle n\rangle$ 


$$
\begin{aligned}
& \vdots \\
& \tilde{\theta}_{n+2}=(J / D)^{3} \sin (2 \pi / p)+\mathcal{O}\left(1 / D^{4}\right) \\
& \tilde{\theta}_{n+1}=(J / D)^{2} \sin (2 \pi / p)+\mathcal{O}\left(1 / D^{3}\right) \\
& \tilde{\theta}_{n}=(J / D) \sin (2 \pi / p)+\mathcal{O}\left(1 / D^{2}\right) \\
& \tilde{\theta}_{n-1}=-J / D \sin (2 \pi / p)+\mathcal{O}\left(H / D, 1 / D^{2}\right) \\
& \tilde{\theta}_{n-2}=-(J / D)^{2} \sin (2 \pi / p)+\mathcal{O}\left(H / D, 1 / D^{3}\right) \\
& \vdots \\
& \tilde{\theta}_{1}=-(J / D)^{n-1} \sin (2 \pi / p)+\mathcal{O}\left(H / D, 1 / D^{n}\right)
\end{aligned}
$$

The final result for the interface phase boundaries derived below will demonstrate that it is consistent to neglect tems $\mathcal{O}(H / D)$ in (6). Note that for $p=2$, for $D$ large, the spins do not lower their energy by canting.

Using the harmonic approximation (3) the energy differences $E_{\langle n\rangle}-E_{\langle n-1\rangle}$ between neighbouring interface states can be calculated. Let $\langle n-1\rangle$ have spins $\left\{\alpha_{i}\right\}$ with $\alpha_{1}$ the surface spin and $\langle n\rangle$ have spins $\left\{\beta_{i}\right\}$ with $\beta_{0}$ the surface spin. Then in both cases the interface lies between $i=n-1$ and $i=n$ and $\alpha_{i}^{0} \equiv \beta_{i}^{0}$. Using this labelling and the recursion equations (5) some algebra leads to the result

$$
\begin{array}{lc}
E_{\langle n\rangle}-E_{\langle n-1\rangle}=-H\{\cos (2 \pi / p)-1\}+H \tilde{\beta}_{1} \sin \beta_{1}^{0} / 2-J \tilde{\beta}_{1} \tilde{\alpha}_{2} / 2 & n \geq 3 \\
E_{\langle 2\rangle}-E_{\langle 1\rangle}=-H\{\cos (2 \pi / p)-1\}+H \tilde{\beta}_{1} \sin \beta_{1}^{0} / 2-J \cos (2 \pi / p) \tilde{\beta}_{1} \tilde{\alpha}_{2} / 2+J \sin (2 \pi / p) \tilde{\beta}_{1} / 2
\end{array}
$$

These formulae are exact for the quadratic Hamiltonian (3). Higher order terms in the full Hamiltonian (11) appear as higher order corrections.

Substituting in the values for the surface spins from (6) gives, for $n \geq 2$,

$$
E_{\langle n\rangle}-E_{\langle n-1\rangle}=-H(\cos (2 \pi / p)-1)-J^{2 n-2} \sin ^{2}(2 \pi / p) /\left(2 D^{2 n-3}\right)+\mathcal{O}\left(1 / D^{2 n-2}\right)
$$

It follows immediately from (9) that the boundary between phases $\langle n-1\rangle$ and $\langle n\rangle$ is given to leading order by 


$$
H_{\langle n-1\rangle:\langle n\rangle}=J^{2 n-2} \sin ^{2}(2 \pi / p)\left\{2(1-\cos (2 \pi / p)) D^{2 n-3}\right\}^{-1}
$$

indicating that the unbinding proceeds via an infinite series of phases $\langle n\rangle$ of widths $\mathcal{O}\left(1 / D^{2 n-3}\right)$

These results were confirmed numerically for the case $p=3$ by studying iteratively the equations which minimize the energy (1). The numerical approach allowed us to obtain the interface phase diagram for all values of $D$ which is shown in Figure 1. The first order boundaries between the different interface phases end at a series of critical end points at

$$
\begin{array}{lll}
\langle 1\rangle:\langle 2\rangle & D_{1,2}^{*}=1.1268 \pm 0.0003 & H_{1,2}^{*}=0.2566 \pm 0.0009 \\
\langle 2\rangle:\langle 3\rangle & D_{2,3}^{*}=0.9360 \pm 0.0003 & H_{2,3}^{*}=0.04357 \pm 0.00001 \\
\langle 3\rangle:\langle 4\rangle & D_{3,4}^{*}=0.7281 \pm 0.0005 & H_{3,4}^{*}=0.01029 \pm 0.00004 \\
\langle 4\rangle:\langle 5\rangle & D_{4,5}^{*}=0.5931 \pm 0.0003 & H_{4,5}^{*}=0.00295 \pm 0.00002
\end{array}
$$

These were identified as the points where both the energy $E$ and its partial derivative with respect to $H$ become the same in the two phases. Assuming that $D_{n, n+1}^{*}$ and $H_{n, n+1}^{*}$ have a power law dependence on $n,\left(D_{\infty}^{*}, H_{\infty}^{*}\right)=(0,0)$ is consistent with the data. However, it was only possible to obtain results for $n \leq 5$, and so we cannot be confident that this is the true asymptotic behaviour.

For $D=0$ the interface shape varies continuously from being a domain wall at the surface for $H$ large to a uniform spiral for $H=0$.

To summarise, we have shown that the softening of discrete spins provides a mechanism, somewhat analogous to temperature, which can stabilise interface layering transitions. An expansion in inverse spin anisotropy was used to demonstrate that an infinite number of such transitions exist at large $D$. Because the transitions take place at zero temperature it was possible to obtain good numerical estimates of the end points of the first order transition lines. 
The model we describe is widely used in the theoretical description of magnetism in the rare-earth metals and compounds [5, 6]. The results presented here may be of relevance to attempts to model mutilayers comprised of these compounds [7]. Moreover, we hope that the system will have theoretical applicability in that it represents one of the simplest models where expansion about a multiphase point is feasible. Aubry [8] has recently pointed out that such multiphase points (which he calls anti-integrable limits) also exist in electronic systems and arrays of non-linear coupled oscillators. It may be possible to use extensions of the technique presented here to treat these systems.

\section{Aknowledgements}

We are grateful to Dr. F. Seno for useful discussions. JMY aknowledges support from an E.P.S.R.C. Advanced Fellowship and CM from an E.P.S.R.C. studentship and from Fondazione "A. Della Riccia", Firenze. 


\section{REFERENCES}

[1] M. J. de Oliveira and R. B. Griffiths, Surf. Sci., B71, 687 (1978)

[2] R. Pandit, M. Schick and M. Wortis, Phys. Rev. B26, 5112 (1982)

[3] P. M. Duxbury and J. M. Yeomans, J. Phys. A18, L983 (1985)

[4] F. Seno and J. M. Yeomans, Oxford University preprint (1994)

[5] J. Jensen and A. R. Mackintosh in Rare Earth Magnetism, Oxford University Press, Oxford (1991)

[6] J. Jensen and A.R. Mackintosh, Phys. Rev. Lett. 64, 2699 (1990)

[7] C. F. Maikrzak, J. Kwo, M Hong, Y. Yafet, D. Gibbs, C. L. Chien, J. Bohr, Adv. Phys. 40, $99(1991)$

[8] S. Aubry, Physica D71, 196 (1994)

\section{Figure Caption}

Fig 1: Phase diagram of the classical X-Y model in a magnetic field, $H$, with 3 -fold spin anisotropy, $D$, and an imposed interface. There are an infinite number of interface layering transitions. The first order boundaries between them terminate in critical end points. 